\title{
Successful treatment of two consecutive cases of pulmonary pleomorphic carcinoma with platinum chemotherapy
}

\author{
DAISUKE KATO ${ }^{1}$, YUICHI CHIHARA ${ }^{1}$, TOMOYUKI SHIRASE ${ }^{2}$, \\ TAMAKI TAKAHASHI ${ }^{1}$, KEN-ICHI TAKAHASHI ${ }^{1}$ and NAOKI SAKAI ${ }^{1}$ \\ ${ }^{1}$ Respiratory Disease Center and ${ }^{2}$ Department of Pathology, Otsu Red Cross Hospital, Otsu, Shiga 520-8511, Japan
}

Received October 25, 2014; Accepted August 5, 2015

DOI: $10.3892 / 01.2015 .3678$

\begin{abstract}
Pulmonary pleomorphic carcinoma (PPC) is a rare pulmonary malignant tumor that has a more aggressive clinical course and a poorer prognosis compared with non-small cell lung cancer (NSCLC) due to its resistance to chemotherapy and radiotherapy. In patients with advanced or relapsed PPC, it has been reported that the response rate to chemotherapy regimens known to be effective in patients with NSCLC is only $0-17 \%$. The present study reports the cases of two consecutive patients with advanced PPC who exhibited marked responses to chemotherapy with carboplatin plus paclitaxel chemotherapy and long-term survival without tumor progression. This suggests that carboplatin plus paclitaxel chemotherapy is a good option for the treatment of advanced PPC.
\end{abstract}

\section{Introduction}

Pulmonary pleomorphic carcinoma (PPC) is a rare primary malignant tumor in which carcinomatous and sarcomatous elements occur. The occurrence of the disease is extremely rare, accounting for only $0.1-0.4 \%$ of all reported malignant lung tumors (1-4). Patients with PPC are reported to be predominantly male smokers with a mean age of 66 years (5-8). PPC generally exhibits an aggressive clinical course, and has a tendency to grow rapidly and invade adjacent structures in the early stage. The recurrence after surgical treatment is common, and systemic metastases are frequently observed in patients with PPC (8). The prognosis of PPC is significantly worse than the majority of other subsets of non-small cell lung cancer (NSCLC) due to its poorer response to chemotherapy and radiotherapy. The response rate to chemotherapy regimens commonly used for NSCLC is in the range of $0-17 \%(2,9)$ and the median survival reported for patients with PPC was

Correspondence to: Dr Yuichi Chihara, Respiratory Disease Center, Otsu Red Cross Hospital, 1-1-35 Nagara, Otsu, Shiga 520-8511, Japan

E-mail: ro3107@kuhp.kyoto-u.ac.jp

Key words: pulmonary pleomorphic carcinoma, chemotherapy, taxanes
5-10 months $(1,2,9)$. The present study reports two cases of advanced PPC that were successfully treated with carboplatin plus paclitaxel chemotherapy and/or radiotherapy.

\section{Case report}

Case 1. A 66-year-old male was admitted to the Otsu Red Cross Hospital (Otsu, Shiga, Japan) in May 2013, due to a persistent cough and dyspnea that had persisted for 10 days. The patient had previously undergone a surgical resection of the colon due to a gastrointestinal stromal tumor. The patient had also smoked two packs of cigarettes per day for 37 years. A performance status score of 1, according to the Eastern Cooperative Oncology Group (ECOG) scale (10), was recorded. Computed tomography (CT) of the chest showed a large tumor, measuring 100x80 mm, in the right lower lobe of the lung, with pleural effusion. An ultrasonography-guided percutaneous lung biopsy was performed, and the tumor specimen consisted of epithelioid and sarcomatous cells (Fig. 1A). On immunohistochemical staining, pankeratin was diffusely positive in the epithelioid and sarcomatous components (Fig. 1B). The patient was diagnosed with PPC based on the morphological findings of the tumor cells admixed with sarcomatoid and epithelioid cells. There was no evidence of metastasis to the other organs, corresponding to a diagnosis of clinical stage IV (cT4N0M1a) in the TNM classification (11). Subsequent to a thoracentesis, systemic chemotherapy was administered consisting of carboplatin [area under the curve (AUC), 6] and paclitaxel $\left(200 \mathrm{mg} / \mathrm{m}^{2}\right)$ on the first day by intravenous administration every three weeks for four cycles. Following chemotherapy, the tumor was markedly reduced in size from 100x80 to $75 \times 22 \mathrm{~mm}$, with disappearance of pleural effusions on chest CT (Fig. 2A and B), indicating that a partial remission was achieved. Mild anemia (grade 2), moderate leukopenia (grade 3) and peripheral neuropathy (grade 3) developed during carboplatin plus paclitaxel chemotherapy, but were not accompanied by any infectious complications, and the patient's performance status improved from 1 to 0 . The patient is currently alive with no tumor progression at 12 months post-chemotherapy.

Case 2. A 69-year-old male presented with abnormal shadows in the left middle lung field on a chest X-ray that was taken during a routine health check, and was admitted to the Otsu 

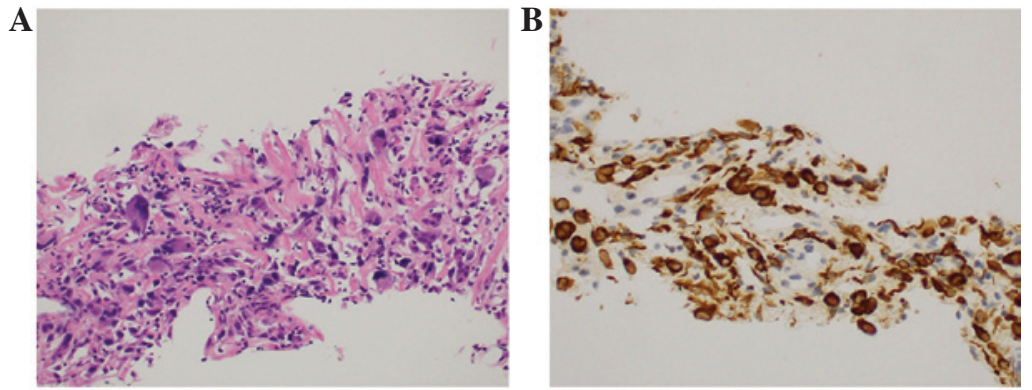

Figure 1.Case 1: (A) Tumor cells consisting of epithelioid and sarcomatous cells (hematoxylin and eosin staining; magnification, $\mathrm{x} 400$ ). (B) Immunohistochemical staining showing diffusely positivity for pankeratin in the epithelioid and sarcomatous components.
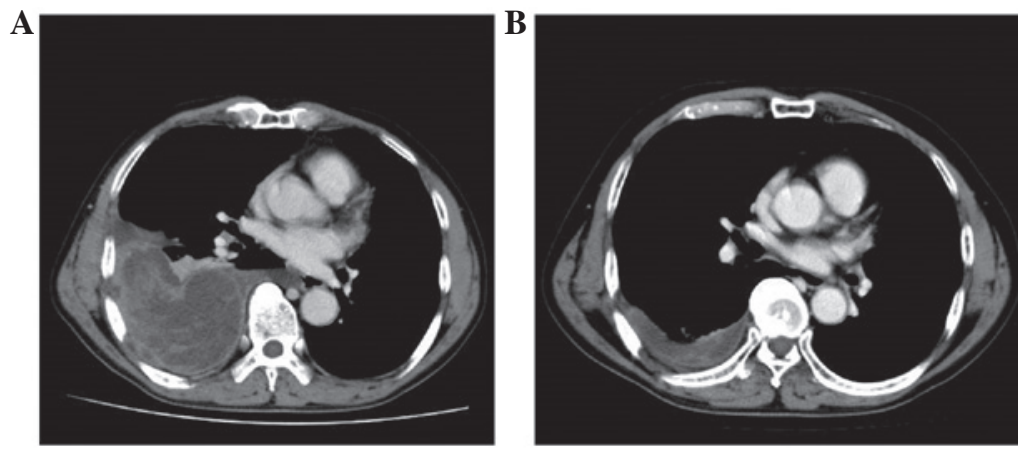

Figure 2. Case 1: Chest CT (A) prior to and (B) following four cycles of carboplatin and paclitaxel chemotherapy. Subsequent to chemotherapy, the tumor size was markedly reduced, with disappearance of the pleural effusions on chest CT. A partial remission was achieved. CT, computed tomography.
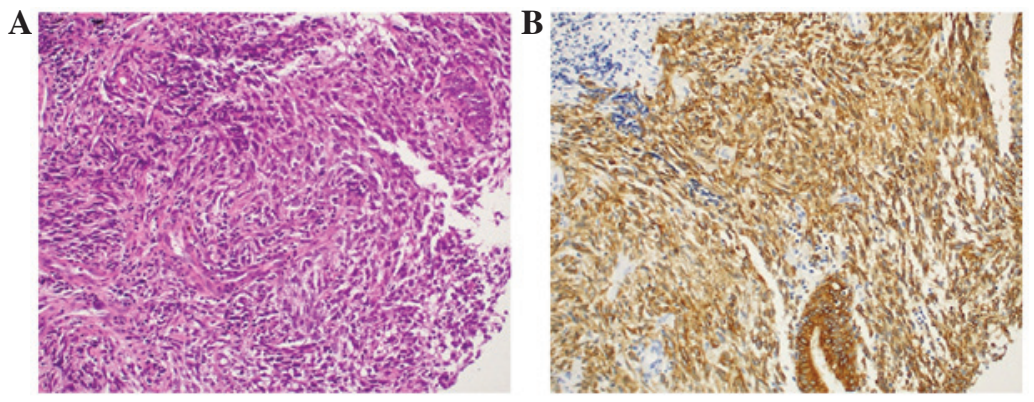

Figure 3. Case 2: (A) Tumor cells exhibiting a biphasic appearance with epithelioid and spindle cell sarcomatous components (hematoxylin and eosin staining; magnification, $\mathrm{x} 400$ ). (B) Positive reaction of the antibody against pankeratin in the epithelioid and spindle cell sarcomatous components.
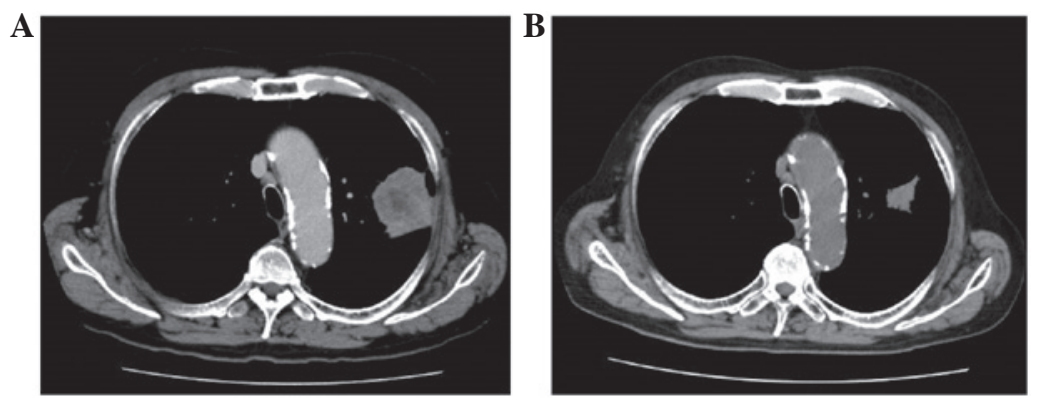

Figure 4. Case 2: Chest CT (A) prior to and (B) following three cycles of carboplatin and paclitaxel chemotherapy plus concurrent radiation therapy. Subsequent to chemotherapy, the tumor was markedly reduced in size on chest CT, and a partial remission was achieved. CT, computed tomography.

Red Cross Hospital (Otsu, Shiga, Japan) in September 2012. The patient had a history of chronic obstructive pulmonary disease and was a smoker of one pack of cigarettes per day for the last 50 years. The ECOG performance status was 0 . CT of the chest showed a lung mass, measuring $54 \times 42 \mathrm{~mm}$, in the left upper lobe, with enlargement of the ipsilateral hilar 
lymph node in addition to emphysema. A transbronchial lung biopsy was performed, and the tumor cells exhibited a biphasic appearance, with epithelioid and spindle cell sarcomatous components (Fig. 3A). Antibodies against pankeratin were reactive in the epithelioid and spindle cell sarcomatous components (Fig. 3B), while antibodies against c-kit, carcinoembryonic antigen, thyroid transcription factor-1 and D2-40 were non-reactive. As a result, a diagnosis of PPC was made. $18^{\mathrm{F}}$-fluorodeoxyglucose positron emission tomography revealed high uptake in the left lung tumor, ipsilateral hilar lymph node and contralateral mediastinal lymph nodes. There was no evidence of metastasis to the other organs, corresponding to a diagnosis of clinical stage IIIB (cT2aN3M0) in the TNM classification (11). The patient received carboplatin (AUC, 6) and paclitaxel $\left(200 \mathrm{mg} / \mathrm{m}^{2}\right)$ every three weeks for three cycles plus concurrent radiation therapy (60 Gy in 30 daily fractions) over a period of 6 weeks. A marked reduction in tumor size from $54 \times 42$ to $18 \times 19 \mathrm{~mm}$ was noted following chemoradiotherapy (Fig. 4A and B), and a partial remission was achieved. During chemoradiotherapy, tolerable myelosuppression and neuropathy (grade 2 anemia and leukopenia, and grade 3 neutropenia and peripheral neuropathy) occurred. The patient is currently alive with no tumor progression at 21 months post-chemoradiotherapy. The two patients gave written informed consent to participate in this clinical study.

\section{Discussion}

PPC was first introduced in the third edition of the World Health Organization (WHO) classifications (12), grouped under the category of carcinomas with pleomorphic, sarcomatoid or sarcomatous elements.PPC is defined as a poorly-differentiated NSCLC with $\geq 10 \%$ giant and/or spindle cell components, or as a carcinoma comprised completely of giant and spindle cells in the new WHO Classification of Lung Cancers (13). PPC is believed to be more aggressive and exhibit a poorer prognosis than ordinary lung carcinomas $(1,3,14)$. There are few studies with regard to the chemotherapy of PPC, and its clinical and pathological characteristics therefore remain unclear. In patients with advanced or relapsed PPC, the response rate to chemotherapy regimens that are known to be effective in patients with NSCLC is only $0-17 \%(2,9)$. A previous study reported the cases of two patients with advanced PPC who showed a partial remission to chemotherapy with doxorubicin, ifosfamide and dacarbazine. However these two patients experienced early relapses following cessation of this combination chemotherapy (15).

The chemosensitivity of surgically-resected PPC has been evaluated using the histoculture drug response assay in a previous study (16). The study demonstrated that taxanes showed a good sensitivity in PPC. Therefore, it was conceivable that PPC may respond to chemotherapy that included taxanes. In the present study, two consecutive patients showed marked responses to carboplatin plus paclitaxel chemotherapy and/or radiotherapy, and experienced long-term survival without tumor progression following therapy. In patients with
PPC, it has also been reported that a tumor size of $>5 \mathrm{~cm}$, a clinical stage of greater than stage I and lymph node involvement confer a significantly poor prognosis (5). Although the two present patients exhibited these poor prognostic factors, they experienced long-term survival. The present results suggest that carboplatin plus paclitaxel chemotherapy and/or radiotherapy may have an important role in the treatment of patients with advanced PPC.

In conclusion, the present study reported two cases in which carboplatin and paclitaxel were markedly effective for PPC. The success with these two cases further suggests that a multi-institutional study to evaluate treatment with carboplatin and paclitaxel is warranted.

\section{References}

1. Fishback NF, Travis WD, Moran CA, et al: Pleomorphic (spindle/ giant cell) carcinoma of the lung. A clinicopathologic correlation of 78 cases. Cancer 73: 2936-2945, 1994.

2. Bae HM, Min HS, Lee SH, et al: Palliative chemotherapy for pulmonary pleomorphic carcinoma. Lung Cancer 58: 112-115, 2007.

3. Park JS, Lee Y, Han J, et al: Clinicopathologic outcomes of curative resection for sarcomatoid carcinoma of the Lung. Oncology 81: 206-213, 2011.

4. Yendamuri S, Caty L, Pine M, et al: Outcomes of sarcomatoid carcinoma of the lung: A Surveillance, Epidemiology and End Results Database analysis. Surgery 152: 397-402, 2012.

5. Rossi G, Cavazza A, Sturm N, et al: Pulmonary carcinomas with pleomorphic, sarcomatoid, or sarcomatous elements: A clinicopathologic and immunohistochemical study of 75 cases. Am J Surg Pathol 27: 311-324, 2003.

6. Yuki T, Sakuma T, Ohbayashi C, et al: Pleomorphic carcinoma of the lung: A surgical outcome. J Thorac Cardiovasc Surg 134: 399-404, 2007.

7. Mochizuki T, Ishii G, Nagai K, et al: Pleomorphic carcinoma of the lung: Clinicopathologic characteristics of 70 cases. Am J Surg Pathol 32: 1727-1735, 2008.

8. Ito K, Oizumi S, Fukumoto S, et al: Clinical characteristics of pleomorphic carcinoma of the lung. Lung Cancer 68: 204-210, 2010.

9. Hong JY, Choi MK, Uhm JE, et al: The role of palliative chemotherapy for advanced pulmonary pleomorphic carcinoma. Med Oncol 26: 287-291, 2009.

10. Oken MM, Creech RH, Tormey DC, et al: Toxicity and response criteria of the Eastern Cooperative Oncology Group. Am J Clin Oncol 5: 649-655, 1982.

11. Goldstraw P, Crowley J, Chansky K, et al: Proposals for the revision of the TNM stage groupings in the forthcoming (seventh) edition of the TNM Classification of malignant tumours. J Thorac Oncol 2: 706-14, 2007.

12. Travis WD, Colby TV, Corrin B, et al: Histological Typing of Lung Tumors and Pleural Tumors. Third edition. Springer Verlag, Berlin, 1999

13. Brambilla E, Travis WD, Colby TV, Corrin B and Shimosato Y: The new World Health Organization classification of lung tumors. Eur Respir J 18: 1059-1068, 2001.

14. Shannon RL, Ro JY, Grignon DJ, et al: Sarcomatoid carcinoma of the prostate. A clinicopathologic study of 12 patients. Cancer 69: 2676-2682, 1992.

15. Lee KW, Kim YJ, Kim JH, et al: Two consecutive cases of platinum-refractory pulmonary pleomorphic carcinoma that showed dramatic responses to MAID (mesna, doxorubicin, ifosfamide and dacarbazine) chemotherapy. Jpn J Clin Oncol 41: 430-433, 2011.

16. Kawano R, Hino H, Hoshino T, et al: Clinicopathological investigation of seven resected cases of pleomorphic carcinoma of the lung and the results of their chemosensitivity tests. Haigan (Jpn J Lung Cancer) 48: 106-111, 2008 (In Japanese). 